\title{
Editorial \\ The relationship between cancer and rheumatoid arthritis: still a large research agenda
}

\section{Thorvardur Love and Daniel H Solomon}

Division of Rheumatology, Immunology, and Allergy, Brigham and Women's Hospital, Harvard Medical School, Francis Street, Boston, MA 02115, USA

Corresponding author: Daniel H Solomon, dsolomon@partners.org

Published: 14 May 2008

Arthritis Research \& Therapy 2008, 10:109 (doi:10.1186/ar2417)

This article is online at http://arthritis-research.com/content/10/3/109

(c) 2008 BioMed Central Ltd

See related research by Smitten et al., http://arthritis-research.com/content/10/2/R45

\begin{abstract}
The association between rheumatoid arthritis (RA) and malignancies has received increased attention in recent years. Reports suggesting that tumor necrosis factor blockers might elevate the risk of malignancy in RA patients have prompted researchers to look at the incidence of malignancies in all RA patients. In a recent issue of Arthritis Research \& Therapy, Smitten and colleagues suggest that previous reports of a standardized incidence ratio close to one for malignancies in RA may reflect an increased risk for some site-specific malignancies and a reduced risk for others. Here we discuss these findings and suggest what issues could be addressed in future studies.
\end{abstract}

In a recent issue of Arthritis Research \& Therapy, Smitten and colleagues [1] reported the results of a meta-analysis of studies looking at the relative risk of malignancies in rheumatoid arthritis (RA) patients compared to the general population, as expressed by a standardized incidence ratio (SIR). While their finding of a SIR of 1.05 for all malignancies in this group supports previous studies, they report SIRs for lymphoma of 2.08 and lung cancer of 1.63 , and decreased relative risks for colorectal cancer of 0.77 and breast cancer of 0.84 . These results suggest that the previously reported SIR close to 1 for all malignancies in RA patients [2] may be explained in part by an increased relative risk for some site-specific malignancies and a reduction in the relative risk for others.

When attempting to separate the reported association between malignancy and RA, the subject of the review by Smitten and colleagues, from the association reported for tumor necrosis factor (TNF) blockers [3], it is important to avoid bias introduced by different patterns of drug use. Smitten and colleagues did not separately analyze TNF blocker treated patients, with 4,917 RA patients from studies on TNF blockers included with other studies for the main analysis. However, given that these two groups of patients on
TNF blockers represent only $2.2 \%$ of the overall cancer group and $2.9 \%$ of the lymphoma group, it seems reasonable to conclude that these patients did not have a large effect on the overall relative risks.

A study by Baecklund and colleagues [4] suggested that there is a relationship between the level of inflammation in RA patients and their risk of developing lymphoma. This strengthens the hypothesis that the association observed between TNF blockers and lymphomas is a consequence of severe RA patients being more likely to receive such drugs. It is also possible that TNF blockers may abolish a TNF-driven mechanism that keeps an indolent lymphoma in check. Should such a mechanism exist, one would expect a spike of lymphomas in the early period of drug use, with an incidence trending down and settling at a rate higher than the baseline seen in RA patients but below the initial spike. Long-term studies of lymphoma incidence in patients treated with TNF blockers are needed to determine if such a pattern will emerge over time. Another possibility is that prolonged TNF blockade will result in a decrease in chronic inflammation, thereby lowering the risk of lymphoma in RA patients in the long-term. Both of these models may turn out to be true, as they are not mutually exclusive.

The discussion by Smitten and colleagues provides an excellent summary of potential explanations for the variable risk across different site-specific malignancies in RA patients. As Baecklund and colleagues' work suggests, ongoing inflammation may, over time, increase the risk of malignant transformation in cells of the immune system, thereby increasing the rates of lymphomas [4]. Similarly, the increased risk for lung cancer may be explained by chronic lung inflammation in RA, but the fact that smoking is a risk factor for both RA and lung cancer may also contribute to the

$\mathrm{RA}=$ rheumatoid arthritis; SIR = standardized incidence ratio; $\mathrm{TNF}=$ tumor necrosis factor. 
observed elevated risk. Increased NSAID (non-steroidal antiinflammatory drug) use may be a factor in protecting RA patients from colon cancer. This possibility is suggested by results of the APPROVE trial, which showed rofecoxib reduces the recurrence of adenomatous polyps as well as the risk of advanced adenomas at one and three years after randomization [5]. Use of immune modulating therapies in RA may also underlie the observed reduction in breast cancer incidence, but the mechanism is not clear.

The important meta-analysis presented by Smitten and colleagues pushes our understanding of malignancy in RA forward but raises many important questions for future research. First, as much of the literature they cite (and most of the patients included in the meta-analysis) is from Sweden, one might ask whether similar patterns of risk would be observed in patients from other ethnic backgrounds. Comparing the Swedish results with those of other studies might give an insight into whether different ethnic backgrounds influence the risk for malignancies in RA patients, thereby suggesting a genetic link between these diseases. Second, in the case of lung cancer it would be very useful to improve the control for potential confounding introduced by tobacco use. This might be achieved with better long-term tobacco histories. Third, as we learn to subtype RA, it would be important to look at the relationship between anti-cyclic citrullinated peptide antibody status and malignancy. Is the risk of lymphoma primarily seen in certain subgroups? This risk might be magnified by specific genetic risk alleles (for example, shared epitope, PTPN22, STAT4). Finally, the clinical relevance of these data regarding RA and malignancy is unclear. Should clinicians screen RA patients differently than typical patients?

\section{Competing interests}

The authors declare that they have no competing interests.

\section{Acknowledgements}

DHS receives salary support from the $\mathrm{NIH}$ (AR 047782 and AR055989).

\section{References}

1. Smitten AL, Simon TA, Hochberg MC, Suissa S: A meta-analysis of the incidence of malignancy in adult patients with rheumatoid arthritis. Arthritis Res Ther 2008, 10:R45.

2. Chakravarty EF, Genovese MC: Associations between rheumatoid arthritis and malignancy. Rheum Dis Clin North Am 2004, 30:271-284, vi.

3. Bongartz T, Sutton AJ, Sweeting MJ, Buchan I, Matteson EL, Montori V: Anti-TNF antibody therapy in rheumatoid arthritis and the risk of serious infections and malignancies: systematic review and meta-analysis of rare harmful effects in randomized controlled trials. JAMA 2006, 295:2275-2285.

4. Baecklund E, lliadou A, Askling J, Ekbom A, Backlin C, Granath F, Catrina Al, Rosenquist R, Feltelius N, Sundstrom C, Klareskog L: Association of chronic inflammation, not its treatment, with increased lymphoma risk in rheumatoid arthritis. Arthritis Rheum 2006, 54:692-701.

5. Baron JA, Sandler RS, Bresalier RS, Quan H, Riddell R, Lanas A, Bolognese JA, Oxenius B, Horgan K, Loftus S, Morton DG; APPROVe Trial Investigators: A randomized trial of rofecoxib for the chemoprevention of colorectal adenomas. Gastroenterology 2006, 131:1674-1682. 